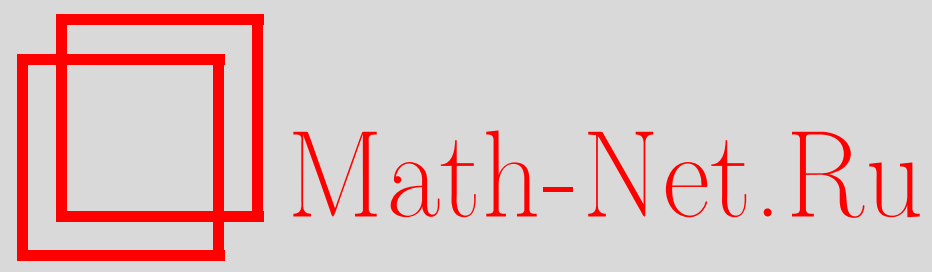

Г. А. Чечкин, Об оценке решений краевых задач в областях с концентрированными массами, периодически расположенными вдоль границы. Случай “легких" масс, Матем. заметки, 2004, том 76, выпуск 6, 928-944

DOI: https://doi.org/10.4213/mzm152

Использование Общероссийского математического портала Math-Net.Ru подразумевает, что вы прочитали и согласны с пользовательским соглашением http://www.mathnet.ru/rus/agreement

Параметры загрузки:

IP : 35.174 .16 .151

26 апреля 2023 г., 14:58:02

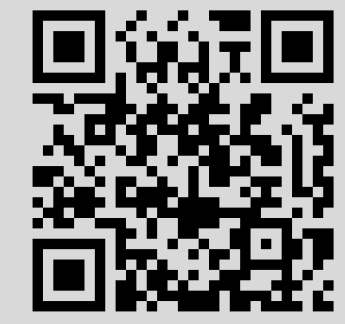




\section{ОБ ОЦЕНКЕ РЕШЕНИЙ КРАЕВЫХ ЗАДАЧ \\ В ОБЛАСТЯХ С КОНЦЕНТРИРОВАННЫМИ МАССАМИ, ПЕРИОДИЧЕСКИ РАСПОЛОЖЕННЫМИ ВДОЛЬ ГРАНИЦЫ. СЛУЧАЙ “ЛЕГКИХ” МАСС}

\section{Г. А. Чечкин}

Мы рассматриваем асимптотическое поведение решений и собственных элементов граничных задач с быстро меняющимся типом граничных условий в области $\Omega \subset \mathbb{R}^{n}$. Плотность, которая зависит от малого параметра $\varepsilon$, имеет порядок $O(1)$ вне мелких включений, где она имеет порядок $O\left((\varepsilon \delta)^{-m}\right)$. Эти области, концентрированные массы диаметра $O(\varepsilon \delta)$, расположены около границы на расстоянии друг от друга порядка $O(\delta)$, где $\delta=\delta(\varepsilon) \rightarrow 0$. Мы ставим условие Дирихле (соответственно Неймана) на участках границы $\partial \Omega$, касающихся (соответственно лежащих вне) концентрированных масс. Получены оценки отклонения решений предельных (усредненных) задач от решений исходной задачи в норме соболевского пространства $W_{2}^{1}$ в случае, когда $m<2$.

Библиография: 50 названий.

Введение. Исследование поведения тел с неоднородной плотностью является одной из интересных задач механики сплошной среды. Первые работы, посвященные этой тематике, начали появляться еще в начале XX века.

В 1913 году Крылов в своей книге [1] рассматривает задачу о колебаниях струны, нагруженной сосредоточенньми массами. Эта работа положила начало глубоким исследованиям в этой области.

С развитием аэродинамики и самолетостроения стало важным изучение устойчивости поведения крыльев и фюзеляжа летательных аппаратов под воздействием вибрации. На крыльях находятся сосредоточенные массы (моторы), фюзеляж также испытывает точечные нагрузки (пассажирские кресла, шасси и т.д.). Такая задача тоже сводится к изучению собственных частот колебаний нагруженной струны (см. приложение к гл. 2 в $[2])$.

Предполагалось, что дополнительная масса $M$ сосредоточена в точке, и рассматривалось предельное поведение решений задачи при стремлении массы к нулю и бесконечности. Особенностью этой модели является точечное прикрепление массы, при этом не учитьваются размеры того множества, где фактически сосредоточена масса.

Работа выполнена при частичной поддержке Российского фонда фундаментальых исследований, грант № 02-01-000693. 
В конще 70-х годов Санчес-Паленсиа рассмотрел задачу, где присоединенная к системе масса сконцентрирована в $\varepsilon$-окрестности внутренней точки, $\varepsilon$ - малый параметр, описьвающий концентрацию и размер массы (см. [3]). В этой работе были использованы методы спектральной теории возмущений.

Другой подход был предложен в работах Олейник [4]-[8]. Базировался этот подход на введении нового основного параметра колебательных систем с локально присоединенными массами - отношения присоединенной массы к массе всей системы. При этом удалось описать локальные колебания системы вблизи сосредоточенной массы. Подробное обоснование модели Олейник-Санчес-Паленсиа, а также анализ размерностей в задаче о спектральных свойствах колебательных систем с присоединенными массами сделал Головатьй (см. [9]).

Рассмотрим общую постановку задачи. Предположим, что колебания механической системы в области $G \subset \mathbb{R}^{n}$ происходят по закону

$$
\begin{cases}\rho(x) \frac{\partial^{2} u}{\partial t^{2}}=P_{0}\left(x, \frac{\partial}{\partial x}\right) u(t, x), & (t, x) \in \mathbb{R}_{+} \times G, \\ B_{0}^{(j)}\left(x, \frac{\partial}{\partial x}\right) u(t, x)=0, & (t, x) \in \mathbb{R}_{+} \times \partial G, \quad j=1, \ldots, l,\end{cases}
$$

где $u(t, x)$ - смещение точки $x \in G$ относительно положения равновесия в момент времени $t$. Здесь $P_{0}, B_{0}^{(j)}, j=1, \ldots, l,-$ дифференциальные операторы, $\rho(x)$ - плотность распределения масс, а функции $u_{0}(x)$ и $u_{1}(x)$ заданы. Примерами таких систем могут служить струна, стержень, мембрана, пластина, упругое тело и т.п. В случае плоской волны $u(t, x)=v(x) e^{i \omega t}$, и мы приходим к спектральной задаче

$$
\begin{cases}P_{0}\left(x, \frac{\partial}{\partial x}\right) v(x)+\omega^{2} \rho(x) v(x)=0, & x \in G, \\ B_{0}^{(j)}\left(x, \frac{\partial}{\partial x}\right) v(x)=0, & x \in \partial G, \quad j=1, \ldots, l .\end{cases}
$$

Пусть имеется следующее распределение масс. Предположим, что в $G$ компактно содержится некое открытое множество $g$ и

$$
\rho(x)=\rho_{G}(x)+\rho_{g}(x) \chi_{g}(x),
$$

где $\rho_{G}(x)>0$ в $\bar{G}, \rho_{g}(x)>0$ в $\bar{g}$, а $\chi_{g}(x)$ - характеристическая функция множества $g$. Рассматривая случай локально сосредоточенной массы, естественно предполагать, что отношение объемов $g$ и $G$ есть мальй параметр $\varepsilon \rightarrow 0$, а отношение средних значений плотностей - большой. Имеем

$$
\frac{|g|^{-1} \int_{g} \rho_{g}(x) d x}{|G|^{-1} \int_{G} \rho_{G}(x) d x}=\varepsilon^{-m}, \quad m \in \mathbb{R}_{+} .
$$

Таким образом, параметр $\varepsilon$ можно считать параметром возмущения задачи. При этом ставится задача изучения влияния сосредоточенной массы на спектр колебательной системы при различных значениях параметра $m$. Приведем некоторые из полученных результатов. 
В [3] Санчес-Паленсиа рассмотрел эту задачу в случае, когда $P_{0}$ - оператор Лапласа с граничными условиями Дирихле и $n=m=3$. В работах Олейник [5]-[8] это сделано для всех $m$ и $n$. В [10] авторы исследовали случай одной концентрированной массы для $P_{0}=d^{2} / d x^{2}$ с граничным условием Дирихле. В случае конечного числа концентрированных масс эта работа была проделана Олейник и Соболевой в [11]. Задача о колебаниях упругого стержня и упругой пластинки с конщентрированными массами изучена в работах Головатого [12], [13]. Случай, когда $P_{0}$ - оператор Лапласа с граничньги условиями Неймана разобран в [14] и [15]. В [16] исследуются первая и третья краевые задачи для оператора Лапласа, $n \geqslant 2$, в случае, когда плотность возмущена конечным числом конщентрированных масс. Асимптотические разложения собственных значений и собственных функций задачи Дирихле для оператора Лапласа получены в [17] и [18]. Случай, когда $P_{0}$ - оператор системы теории упругости рассмотрели Санчес-Паленсиа и Чата в [19]. О колебании мембраны см. [20]. В [21]-[28] рассматривается асимптотика колебаний тела, имеющего много небольших включений большой плотности, расположенных периодически вдоль гранищы. Изучены вопросы асимптотического поведения собственных значений. Аналогичные задачи были рассмотрены в [29]-[31]. В работе [32] рассматривается краевая задача для стационарой системы линейной теории упругости с непериодическими быстро меняюшимися граничными условиями и большим количеством конщентрированньх масс около границы, ее асимптотическое поведение, а также предельное поведение спектра этой краевой задачи. Разобран случай краевой задачи для системы теории упругости, когда предельная задача имеет третье краевое условие на границе области, а плотность включений не слишком велика (значение параметра $m<2$ ). Получены оценки скорости сходимости решения исходной задачи $\mathrm{k}$ решению усредненной, а также поведение собственных элементов такой краевой задачи.

Во всех этих моделях предполагается, что закон колебания груза или уплотнения должен описываться теми же уравнениями, которыми описываются колебания самой системы. В работе [33] рассматривается задача для линейной стационарной системы теории упругости в областях с концентрированными массами. Рассмотрены различные случаи поведения собственных элементов таких задач. В работе рассматривается ситуация, когда включения достаточно жесткие. При этом законы колебания тела и масс различны.

Работа [34] посвящена детальному изучению поведения собственных элементов оператора Лапласа в области с непериодическими "легкими" концентрированными массами.

В настоящей работе рассматривается случай произвольного $n \geqslant 3$ и периодически расположенных масс, даются в соответствующих нормах оценки отклонения (оценки скорости сходимости) решений поставленных задач от решений предельных задач при стремлении малого параметра к нулю, а также аналогичные оценки получены для собственных значений соответствующей спектральной задачи. Базовые оценки работы частично аннонсированы в [35].

1. Постановка задачи. Пусть $\Omega$ - гладкая область в $\mathbb{R}^{n}, n \geqslant 3$, обозначим через $\partial \Omega$ ее границу. Предполагается, что $\partial \Omega=\Gamma_{1} \cup \Gamma_{2}$, гиперплоскость $\Gamma_{2}$ состоит из двух частей, $\Gamma_{\varepsilon}$ и $\gamma_{\varepsilon}$, где $\gamma_{\varepsilon}=\bigcup_{i=1}^{N_{\delta}} \gamma_{\varepsilon}^{i}$. Введем следующее обозначение: $B_{\varepsilon}=\bigcup_{i=1}^{N_{\delta}} B_{\varepsilon}^{i}$ 
- объединение полушаров, находящихся внутри области $\Omega$. Поясним теперь построение. Пусть $B_{\varepsilon}$ - гомотетичное сжатие $\delta B, B^{0}$ - это полушар $\left\{\left(\xi_{1}, \ldots, \xi_{n}\right) \mid \xi_{1}^{2}+\cdots+\xi_{n}^{2}<\varepsilon^{2}\right.$, $\left.\xi_{n}<0\right\}$ в растянутом пространстве $\mathbb{R}^{n}, \xi=x / \delta$,

$$
\gamma_{0}=\left\{\left(\xi_{1}, \ldots, \xi_{n}\right) \mid \xi_{1}^{2}+\cdots+\xi_{n-1}^{2}<\varepsilon^{2}, \xi_{n}=0\right\}
$$

$B$ - область, полученная целочисленньми сдвигами множества $B^{0}$ на гиперплоскости $\left\{\xi_{n}=0\right\}$ с центрами в точках

$$
\widetilde{\xi}_{k}=\left(k_{1}, \ldots, k_{n-1}, 0\right), \quad k_{1}, \ldots, k_{n-1} \in \mathbb{N}
$$

Геометрическая конфигурация на гиперплоскости $\left\{x_{n}=0\right\}$ аналогична описанной в [22], [23], [36] (см. рис. 1). При этом

$$
\gamma_{\varepsilon}=\bar{B}_{\varepsilon} \cap \partial \Omega
$$

Отметим, что рассматривается случай, когда параметр $\delta(\varepsilon)$, определяющий характерное расстояние между участками $\gamma_{\varepsilon}^{i}$ на границе, стремится к нулю при $\varepsilon \rightarrow 0$. Также заметим, что $N_{\delta}=O\left(1 / \delta^{n-1}\right)$.

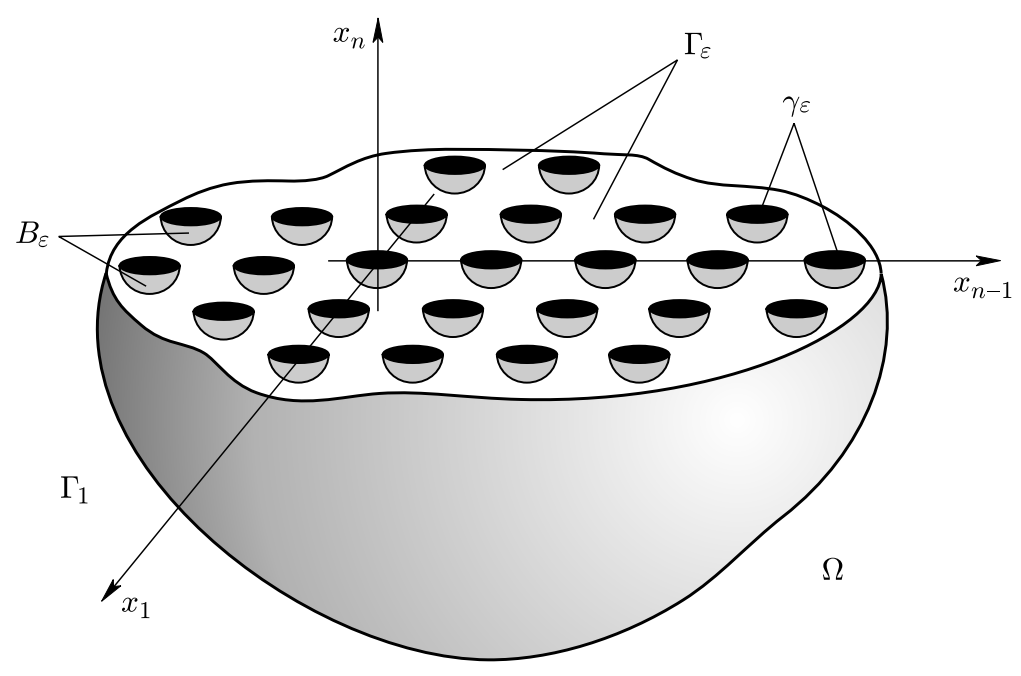

Рис. 1

Рассмотрим следующую спектральную задачу:

$$
\begin{cases}\Delta u_{\varepsilon}^{k}=-\lambda_{\varepsilon}^{k} \rho^{\varepsilon}(x) u_{\varepsilon}^{k} & \text { в } \Omega, \\ u_{\varepsilon}^{k}=0 & \text { на } \Gamma_{1} \cup \gamma_{\varepsilon} \\ \frac{\partial u_{\varepsilon}^{k}}{\partial \nu}=0 & \text { на } \Gamma_{\varepsilon}\end{cases}
$$


где $\nu$ - единичньй вектор внешней нормали к границе $\partial \Omega$,

$$
\rho^{\varepsilon}(x)= \begin{cases}\frac{1}{(\varepsilon \delta)^{m}}, & x \in B_{\varepsilon}, \\ 1, & x \in \Omega \backslash B_{\varepsilon}\end{cases}
$$

Рассматривается случай $m<2$.

Собственные значения $\left\{\lambda_{\varepsilon}^{k}\right\}$ занумерованы в порядке неубывания, т.е.

$$
\lambda_{\varepsilon}^{1} \leqslant \lambda_{\varepsilon}^{2} \leqslant \cdots \leqslant \lambda_{\varepsilon}^{k} \leqslant \cdots,
$$

и повторяются с учетом кратности. При этом

$$
\int_{\Omega} \rho^{\varepsilon}(x) u_{\varepsilon}^{k} u_{\varepsilon}^{l} d x=\delta_{k l} .
$$

Для формулировки теорем нам понадобится следующая величина:

$$
P:=\lim _{\varepsilon \rightarrow 0} \frac{\varepsilon^{n-2}}{\delta(\varepsilon)} .
$$

Обозначим (см. рис. 2)

$$
\begin{aligned}
& D=\left\{\xi \in \mathbb{R}^{n} \mid-\frac{1}{2}<\xi_{i}<\frac{1}{2}, i=1, \ldots, n-1, \xi_{n}<0\right\} \\
& \Sigma=\left\{\xi \in \mathbb{R}^{n} \mid-\frac{1}{2}<\xi_{i}<\frac{1}{2}, i=1, \ldots, n-1, \xi_{n}=0\right\}
\end{aligned}
$$

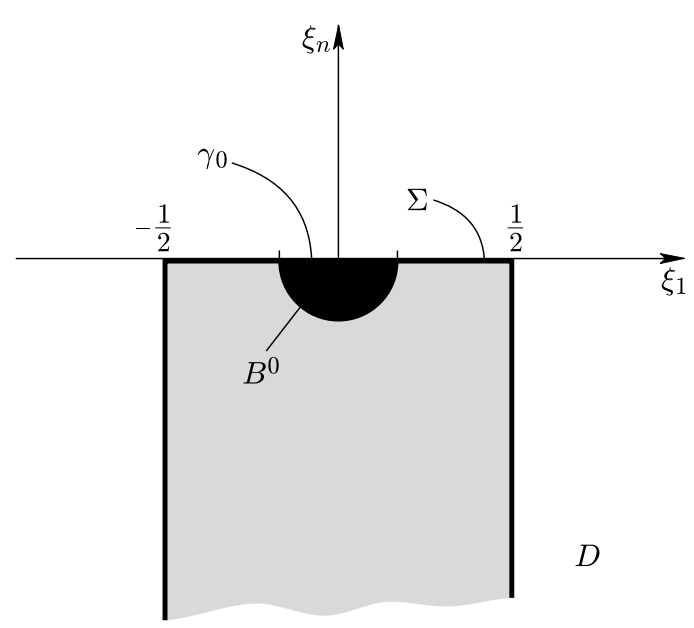

Рис. 2 
Пусть функция $W^{\varepsilon}$, периодическая по переменным $\xi_{1}, \ldots, \xi_{n-1}$, является первой собственной функцией задачи типа Стеклова

$$
\begin{cases}\Delta W^{\varepsilon}=0 & \text { в } D, \\ W^{\varepsilon}=0 & \text { на } \gamma_{0}, \\ \frac{\partial W^{\varepsilon}}{\partial \xi_{n}}=\nu_{\varepsilon} W^{\varepsilon} & \text { на } \Sigma \backslash \gamma_{0} .\end{cases}
$$

Зададим $w_{\varepsilon}$ формулой

$$
w_{\varepsilon}(x)=1+\psi\left(x_{n}\right)\left(W^{\varepsilon}\left(\frac{x}{\delta}\right)-1\right)
$$

и продолжим ее по периодичности. Здесь $\psi(t)$ - гладкая срезающая функция одной переменной, $0 \leqslant \psi \leqslant 1, \psi \equiv 1$ в некоторой достаточно малой окрестности $\Gamma_{2}$. Свойства функции $w_{\varepsilon}$ приведены в предложении в начале следующего пункта и подробно изучены в $[36]$.

Приведем далее основные результаты, которые будут доказаныпозднее. Рассмотрим краевые задачи

$$
\begin{gathered}
\begin{cases}\Delta u^{\varepsilon}=-\rho^{\varepsilon}(x) f^{\varepsilon} & \text { в } \Omega, \\
u^{\varepsilon}=0 & \text { на } \Gamma_{1} \cup \gamma_{\varepsilon}, \\
\frac{\partial u^{\varepsilon}}{\partial \nu}=0 & \text { на } \Gamma_{\varepsilon},\end{cases} \\
\left\{\begin{array}{ll}
\Delta u^{0}=-f^{0}(x) \quad \text { в } \Omega, \\
u^{0}=0 \quad \text { на } \partial \Omega, & P=+\infty, \\
\frac{\partial u^{0}}{\partial \nu}+P \frac{\sigma_{n} c_{\gamma_{0}}}{2} u^{0}=0 & \text { на } \Gamma_{2}, \\
u^{0}=0 & \text { на } \Gamma_{1}
\end{array}\right], P<+\infty,
\end{gathered}
$$

где $\sigma_{n}$ - площадь единичной $n$-мерной сферы, а $c_{\gamma_{0}}:=\operatorname{cap}\left(\gamma_{0}\right)$ - гармоническая емкость $(n-1)$-мерного диска $\gamma_{0}$.

Имеет место

Теорема 1. Если $P<+\infty, u^{\varepsilon} u u^{0}$ - обобщенные решения задач (4) $и$ (5) соответственно, то существует такая константа $K_{1}\left(F, \gamma_{0}, n\right)$, не зависящая от $\varepsilon и \delta$, что для достаточно мальх $\varepsilon$ имеем

$$
\left\|u^{0} w_{\varepsilon}-u^{\varepsilon}\right\|_{H^{1}(\Omega)} \leqslant K_{1}\left(\varepsilon^{(n-2) / 2}+\left|\frac{\varepsilon^{n-2}}{\delta}-P\right|+\varepsilon^{2-m} \delta^{2-m}\right) .
$$

Если $P=+\infty$, то существует $K_{2}\left(f, \gamma_{0}, n\right)$ такое, что

$$
\left\|u^{0} w_{\varepsilon}-u^{\varepsilon}\right\|_{H^{1}(\Omega)} \leqslant K_{2}\left(\varepsilon^{(n-2) / 2}+\frac{\delta^{1 / 2}}{\varepsilon^{(n-2) / 2}}+\varepsilon^{2-m} \delta^{2-m}\right) .
$$


Теперь приведем спектральную задачу

$$
\left\{\begin{array}{l}
\Delta u_{0}^{k}=-\lambda_{0}^{k} u_{0}^{k} \quad \text { в } \Omega, \\
u_{0}^{k}=0 \quad \text { на } \partial \Omega, \quad P=+\infty \\
{\left[\begin{array}{lr}
\frac{\partial u_{0}^{k}}{\partial \nu}+P \frac{\sigma_{n} c_{\gamma_{0}}}{2} u_{0}^{k}=0 & \text { на } \Gamma_{2}, \\
u_{0}^{k}=0 & \text { на } \Gamma_{1}
\end{array}\right], \quad P<+\infty} \\
\int_{\Omega} u_{0}^{k} u_{0}^{l} d x=\delta_{k l}, \quad 0<\lambda_{0}^{1} \leqslant \lambda_{0}^{2} \leqslant \cdots .
\end{array}\right.
$$

Имеет место

ТЕОрема 2. Пусть $\lambda_{0}^{k}, \lambda_{\varepsilon}^{k}$ являются собственными значениями задач (6) и (1) соответственно. Тогда

$$
\begin{array}{ll}
\left|\lambda_{0}^{k}-\lambda_{\varepsilon}^{k}\right| \leqslant C_{k}^{1}\left(\varepsilon^{(n-2) / 2}+\left|\frac{\varepsilon^{n-2}}{\delta}-P\right|+\varepsilon^{2-m} \delta^{2-m}\right), \quad \text { ecлu } \quad P<\infty, \\
\left|\lambda_{0}^{k}-\lambda_{\varepsilon}^{k}\right| \leqslant C_{k}^{2}\left(\varepsilon^{(n-2) / 2}+\frac{\delta^{1 / 2}}{\varepsilon^{(n-2) / 2}}+\varepsilon^{2-m} \delta^{2-m}\right), \quad \text { еслu } \quad P=+\infty,
\end{array}
$$

где постоянние $C_{k}^{1}, C_{k}^{2}$ не зависят от $\varepsilon$.

Если кратность собственного значения $\lambda_{0}^{l}$ задачи (6) равна $r$, т.е. $\lambda_{0}^{l}=\lambda_{0}^{l+1}=$ $\cdots=\lambda_{0}^{l+r}$, то для любой собственной функиии $u_{0}^{l}$ задачи (6), соответствующей собственному значению $\lambda_{0}^{l},\left\|u_{0}\right\|_{L_{2}(\Omega)}=1$, существует линейная комбинация $\overline{u^{\varepsilon}}$ собственных функиий задачи (1), соответствующих собственному значению $\lambda_{\varepsilon}^{l+1}, \ldots, \lambda_{\varepsilon}^{l+r}$, такая, что

$$
\begin{gathered}
\left(\int_{\Omega} \rho^{\varepsilon}(x)\left|\overline{u^{\varepsilon}}-u_{0}^{l}\right|^{2} d x\right)^{1 / 2} \leqslant C_{l}^{1}\left(\varepsilon^{(n-2) / 2}+\left|\frac{\varepsilon^{n-2}}{\delta}-P\right|+\varepsilon^{2-m} \delta^{2-m}\right), \\
e c \Omega u \quad P<\infty, \\
\left(\int_{\Omega} \rho^{\varepsilon}(x)\left|\overline{u^{\varepsilon}}-u_{0}^{l}\right|^{2} d x\right)^{1 / 2} \leqslant C_{l}^{2}\left(\begin{array}{c}
\left.\varepsilon^{(n-2) / 2}+\frac{\delta^{1 / 2}}{\varepsilon^{(n-2) / 2}}+\varepsilon^{2-m} \delta^{2-m}\right), \\
\text { ecлu } \quad P=+\infty,
\end{array}\right.
\end{gathered}
$$

әде постоянные $C_{l}^{1}, C_{l}^{2}$ не зависят от в и $u_{0}^{l}$.

2. Вспомогательные утверждения и предварительные замечания. Мы будем изучать предельное поведение решений спектральной задачи (1) при $\varepsilon$, стремящемся к нулю. Существование и единственность решения $u_{\varepsilon}^{k}$ задачи $(1)$ в пространстве $H^{1}\left(\Omega, \gamma_{\varepsilon}\right)$ может быть доказана на основе леммы Лакса-Мильграма (см., например, [37]). Пространство $H^{1}\left(\Omega, \gamma_{\varepsilon}\right)$ определяем как пополнение множества функций из пространства $C^{\infty}(\bar{\Omega})$, обращаюшихся в нуль в окрестности $\Gamma_{1} \cup \gamma_{\varepsilon}$, по норме

$$
\|u\|_{H^{1}(\Omega)} \equiv\left(\int_{\Omega}\left(u^{2}+|\nabla u|^{2}\right) d x\right)^{1 / 2} .
$$


Пространство $H^{1}\left(D, \gamma_{0}\right)$ - это пополнение по норме

$$
\|u\|_{1} \equiv\left(\int_{D}\left|\nabla_{\xi} u\right|^{2} d \xi+\int_{\Sigma} u^{2} d \widehat{\xi}\right)^{1 / 2}
$$

множества 1 -периодических по $\widehat{\xi} \equiv\left(\xi_{1}, \ldots, \xi_{n-1}\right)$ функций из $C^{\infty}(\bar{D})$, обращающихся в нуль в окрестности $\gamma_{0}$ и обладающих конечным интегралом Дирихле по области $D$.

Пусть

$$
\nu_{\varepsilon}=\inf _{v \in H^{1}\left(D, \gamma_{0}\right) \backslash\{0\}}\left(\frac{\int_{D}\left|\nabla_{\xi} v\right|^{2} d \xi}{\int_{\Sigma} v^{2} d \widehat{\xi}}\right) .
$$

В работе [38] исследовался вопрос поведения собственного значения $\nu_{\varepsilon}$. Там доказана следующая асимптотика (см. формулу (25) работы [38]).

ПРЕДЛОЖЕНИЕ 1. Пусть $\sigma_{n}-$ площадь единичной $n$-мерной сферы, а $c_{\gamma_{0}}-$ гармоническая емкость $\gamma_{0}$. Тогда

$$
\nu_{\varepsilon}=\varepsilon^{n-2} \frac{\sigma_{n}}{2} c_{\gamma_{0}}+o\left(\varepsilon^{n-2}\right) .
$$

В дальнейшем мы воспользуемся результатами этой леммы. Также в дальнейшем будем использовать следующее утверждение из [36].

ПРЕДЛОЖЕНИЕ 2. Существует такая гармоническая в $D$ функиия $W^{\varepsilon}(\xi) \in$ $H^{1}\left(D, \gamma_{0}\right)$, что на ней достигается нижняя грань в (7),

$$
\int_{D}\left|\nabla_{\xi} W^{\varepsilon}\right|^{2} d \xi=\nu_{\varepsilon}, \quad\left\|W^{\varepsilon}\right\|_{L_{2}(\Sigma)}=1
$$

причем граничное условие

$$
\frac{\partial W^{\varepsilon}}{\partial \xi_{n}}=\nu_{\varepsilon} W^{\varepsilon} \quad \text { на } \quad \Sigma \backslash \gamma_{0}
$$

выполняется в следующем смысле:

$$
\int_{\Sigma_{\rho}} \frac{\partial W^{\varepsilon}}{\partial \xi_{n}} v d \widehat{\xi} \rightarrow \nu_{\varepsilon} \int_{\Sigma} W^{\varepsilon} v d \widehat{\xi} \quad n p u \rho \rightarrow 0 \quad \text { для любой } v \in H^{1}\left(D, \gamma_{0}\right) .
$$

Здесь $\Sigma_{\rho}=\left\{\xi \in \mathbb{R}^{n} \mid 0<\xi_{i}<1, i=1, \ldots, n-1, \xi_{n}=-\rho\right\}$.

В работе [36] подробно обсуждается вопрос существования таких функций и их свойства, некоторые из этих свойств могут быть доказаны на основе результатов работ [39] и $[40]$.

Положим

$$
w_{\varepsilon}(x)=1+\psi\left(x_{n}\right)\left(W^{\varepsilon}\left(\frac{x}{\delta}\right)-1\right),
$$

продолжив по периодичности.

Заметим, что ввиду (8)

$$
\frac{\partial w_{\varepsilon}}{\partial x_{n}}=\frac{\nu_{\varepsilon}}{\delta} w_{\varepsilon} \quad \text { на } \quad \Gamma_{\varepsilon} .
$$


ЛЕмма 1. Для функиий $v \in H^{1}\left(\Omega, \gamma_{\varepsilon}\right)$ справедливо следующее неравенство:

$$
\int_{B_{\varepsilon}^{i}} v^{2} d x \leqslant C(\varepsilon \delta)^{2} \int_{B_{\varepsilon}^{i}}|\nabla v|^{2} d x, \quad i=1, \ldots, N_{\delta} .
$$

ДоКАЗАТЕЛЬСтво (см. [22], а также [41]) основано на применении неравенства (см. $[41,(5.3)$, с. 263]) Харди.

Лемма 2. Для последовательности функиий $\left\{v_{\varepsilon}\right\} \in H^{1}\left(\Omega, \gamma_{\varepsilon}\right)$ выполняется следующее равенство:

$$
\lim _{\varepsilon \rightarrow 0}(\varepsilon \delta)^{-2} \int_{B_{\varepsilon}^{i}}\left|v_{\varepsilon}\right|^{2} d x=0,
$$

если $\left\|v_{\varepsilon}\right\|_{H^{1}(\Omega)} \leqslant K$, әде $K$ не зависит от $\varepsilon$.

ДоКАЗАТЕльСТво (аналогично см. [41]). Пусть $v \in H^{1}\left(\Omega, \gamma_{\varepsilon}\right)$. Для любого числа $\alpha>0$ существует функция $v_{\alpha} \in C^{\infty}\left(\Omega, \gamma_{\varepsilon}\right)$ такая, что $C^{1 / 2}\left\|v-v_{\alpha}\right\|_{H^{1}(\Omega)}<\alpha$, где $C-$ постоянная из формулы (10). Теперь, используя оценку (10) для $v-v_{\alpha}$, получаем

$$
\begin{aligned}
& \left(\int_{B_{\varepsilon}^{i}}(\varepsilon \delta)^{-2}|v|^{2} d x\right)^{1 / 2} \leqslant\left(\int_{B_{\varepsilon}^{i}}(\varepsilon \delta)^{-2}\left|v-v_{\alpha}\right|^{2} d x\right)^{1 / 2}+\left(\int_{B_{\varepsilon}^{i}}(\varepsilon \delta)^{-2}\left|v_{\alpha}\right|^{2} d x\right)^{1 / 2} \\
& \leqslant C^{1 / 2}\left(\int_{\Omega}\left|\nabla\left(v-v_{\alpha}\right)\right|^{2} d x\right)^{1 / 2}+C_{\alpha}(\varepsilon \delta)^{n / 2-1} \leqslant \alpha+C_{\alpha}(\varepsilon \delta)^{n / 2-1} .
\end{aligned}
$$

Так как $n \geqslant 3$, то лемма 2 доказана.

Лемма 3. Пусть $u^{\varepsilon} \in H^{1}\left(\Omega, \gamma_{\varepsilon}\right)$ - решение уравнения

$$
\Delta u^{\varepsilon}=-\rho^{\varepsilon}(x) f^{\varepsilon}(x) \quad \boldsymbol{\theta} \Omega .
$$

Тогда

$$
\int_{\Omega}\left|\nabla u^{\varepsilon}\right|^{2} d x \leqslant C_{1}\left((\varepsilon \delta)^{2-m} \int_{B_{\varepsilon}} \rho^{\varepsilon}(x)\left|f^{\varepsilon}\right|^{2} d x+\int_{\Omega}\left|f^{\varepsilon}\right|^{2} d x\right) .
$$

ДокАЗАТЕльСтво. Из интегрального тождества, учитывая лемму 1 и неравенство типа Фридрихса (см., например, [42]-[45]), получаем

$$
\begin{gathered}
\left\|\nabla u^{\varepsilon}\right\|_{L_{2}(\Omega)}^{2}=\int_{\Omega}\left|\nabla u^{\varepsilon}\right|^{2} d x=\int_{\Omega} \rho^{\varepsilon} f^{\varepsilon} u^{\varepsilon} d x=\int_{\Omega \backslash B_{\varepsilon}} f^{\varepsilon} u^{\varepsilon} d x+(\varepsilon \delta)^{-m} \int_{B_{\varepsilon}} f^{\varepsilon} u^{\varepsilon} d x \\
\leqslant\left(\int_{\Omega}\left(f^{\varepsilon}\right)^{2} d x\right)^{1 / 2}\left(\int_{\Omega}\left(u^{\varepsilon}\right)^{2} d x\right)^{1 / 2}+(\varepsilon \delta)^{-m}\left(\int_{B_{\varepsilon}}\left(f^{\varepsilon}\right)^{2} d x\right)^{1 / 2}\left(\int_{B_{\varepsilon}}\left(u^{\varepsilon}\right)^{2} d x\right)^{1 / 2} \\
\leqslant C_{2}\left(\int_{\Omega}\left(f^{\varepsilon}\right)^{2} d x\right)^{1 / 2}\left\|\nabla u^{\varepsilon}\right\|_{L_{2}(\Omega)}+C_{3}(\varepsilon \delta)^{-m+1}\left(\int_{B_{\varepsilon}}\left(f^{\varepsilon}\right)^{2} d x\right)^{1 / 2}\left\|\nabla u^{\varepsilon}\right\|_{L_{2}\left(B_{\varepsilon}\right)} .
\end{gathered}
$$

Отсюда вытекает непосредственно наше неравенство. Лемма 3 доказана. 
3. Основные утверждения. Поведение собственных значений и собственных функций задачи (1) будем изучать на основе общей схемы, предложенной в [41].

Пусть $H_{\varepsilon}, H_{0}$ - сепарабельные гильбертовы пространства со скалярным произведением $(u, v)_{\varepsilon},(u, v)_{0}$ и нормами $\|u\|_{\varepsilon},\|u\|_{0}$ соответственно, $\varepsilon$ - мальй положительный параметр, и пусть $A_{\varepsilon} \in \mathscr{L}\left(H_{\varepsilon}\right), A_{0} \in \mathscr{L}\left(H_{0}\right)$ - линейные непрерывные операторы, причем $\operatorname{Im} A_{0} \subset V \subset H_{0}$; где $V$ - линейное подпространство в $H_{0}$.

Будем предполагать, что вьполнены следующие условия C1-C4.

УСловиЕ С1. Существуют линейные непрерьвные операторы $R_{\varepsilon}: H_{0} \rightarrow H_{\varepsilon}$ такие, что для любой функции $f \in V$ имеем

$$
\left(R_{\varepsilon} f, R_{\varepsilon} f\right)_{\varepsilon} \rightarrow \varkappa(f, f)_{0} \quad \text { при } \varepsilon \rightarrow 0,
$$

где $\varkappa=$ const $>0$ и не зависит от $f$.

УСловиЕ С2. Операторы $A_{\varepsilon}, A_{0}$ являются положительными, компактными и самосопряженньми в $H_{\varepsilon}$ и $H_{0}$ соответственно, причем

$$
\sup _{\varepsilon}\left\|A_{\varepsilon}\right\|_{\mathscr{L}\left(H_{\varepsilon}\right)}<\infty
$$

УСловиЕ С3. Для любой $f \in V$ имеем

$$
\left\|A_{\varepsilon} R_{\varepsilon} f-R_{\varepsilon} A_{0} f\right\|_{\varepsilon} \rightarrow 0 \quad \text { при } \varepsilon \rightarrow 0 .
$$

УСловиЕ С4. Семейство операторов $A_{\varepsilon}$ равномерно компактно в следующем смысле. Из любой последовательности $f^{\varepsilon} \in H_{\varepsilon}$ такой, что $\sup _{\varepsilon}\left\|f^{\varepsilon}\right\|_{\varepsilon}<\infty$, можно выбрать подпоследовательность $f^{\varepsilon^{\prime}}$ и найти функцию $w \in V$ такие, что

$$
\left\|A_{\varepsilon^{\prime}} f^{\varepsilon^{\prime}}-R_{\varepsilon^{\prime}} w\right\|_{\varepsilon^{\prime}} \rightarrow 0 \quad \text { при } \quad \varepsilon^{\prime} \rightarrow 0
$$

Рассмотрим спектральные задачи для операторов $A_{\varepsilon}, A_{0}$ :

$$
\begin{array}{ll}
A_{\varepsilon} u_{\varepsilon}^{k}=\mu_{\varepsilon}^{k} u_{\varepsilon}^{k}, & k \in \mathbb{N}, \quad\left(u_{\varepsilon}^{i}, u_{\varepsilon}^{j}\right)=\delta_{i j}, \\
A_{0} u_{0}^{k}=\mu_{0}^{k} u_{0}^{k}, \quad k \in \mathbb{N}, \quad\left(u_{0}^{i}, u_{0}^{j}\right)=\delta_{i j},
\end{array}
$$

где $\delta_{i j}$ - символ Кронекера, собственные значения $\mu_{\varepsilon}^{k}, \mu_{0}^{k}$ занумерованы в порядке невозрастания, причем каждое собственное значение повторяется столько раз, какова его кратность. 
ТЕоремА (Олейник-Иосифьян-Шамаев). а) Пусть выполнены условия С1-С4. Тогда

$$
\left|\mu_{\varepsilon}^{k}-\mu_{0}^{k}\right| \leqslant M_{\varepsilon} \sup _{f}\left\|A_{\varepsilon} R_{\varepsilon} f-R_{\varepsilon} A_{0} f\right\|_{\varepsilon}, \quad k \in \mathbb{N},
$$

где $\mu_{\varepsilon}^{k}, \mu_{0}^{k}-k$-е собственное значение задач (11), (12) соответственно, верхняя грань берется по всем функииям $f \in N\left(\mu_{0}^{k}, A_{0}\right)=\left\{v \in H_{0}: A_{0} v=\mu_{0}^{k} v\right\}$ таким, что $\|v\|_{0}=1$, постоянная $M_{\varepsilon} \leqslant \mathscr{M}$ при $0<\varepsilon \leqslant 1, u M_{\varepsilon} \rightarrow 1 / \sqrt{\varkappa}$ при $\varepsilon \rightarrow 0, \mathscr{M}$ не зависит от $\varepsilon$.

б) Пусть $k \leqslant 0, l \leqslant 1$ - иелье числа, кратность собственного значения $\mu_{0}^{k+1}$ задачи (12) равна $l$, m.е. $\mu_{0}^{k+1}=\cdots=\mu_{0}^{k+l}$. Тогда для любой $u_{0} \in N\left(\mu_{0}^{k+1}, A_{0}\right)$ существует линейная комбинация $\bar{u}_{\varepsilon}$ собственных функиий $u_{\varepsilon}^{k+1}, \ldots, u_{\varepsilon}^{k+l}$ задачи (11) такая, что

$$
\left\|\bar{u}_{\varepsilon}-R_{\varepsilon} u_{0}\right\|_{\varepsilon} \leqslant M_{k}\left\|A_{\varepsilon} R_{\varepsilon} u_{0}-R_{\varepsilon} A_{0} u_{0}\right\|_{\varepsilon}
$$

где постоянная $M_{k}$ не зависит от $\varepsilon$.

Для того чтобы воспользоваться схемой, нужно подходящим образом ввести пространства $H_{0}, H_{\varepsilon}, V$, операторы $A_{0}, A_{\varepsilon}, R_{\varepsilon}$ и проверить выполнение условий C1-C4.

Для удобства введем такое обозначение:

$$
p:=P \frac{\sigma_{n} c_{\gamma_{0}}}{2}
$$

Обозначим соответственно через $H_{\varepsilon}$ и $H_{0}$ пространство $L_{2}(\Omega)$ со скалярным произведением

$$
\left(f^{\varepsilon}, g^{\varepsilon}\right)_{H_{\varepsilon}} \equiv \int_{\Omega} \rho^{\varepsilon}(x) f^{\varepsilon} g^{\varepsilon} d x, \quad\left(f^{0}, g^{0}\right)_{H_{0}} \equiv \int_{\Omega} f^{0} g^{0} d x
$$

соответственно. В качестве $V$ возьмем пространство $\stackrel{\circ}{H}^{1}(\Omega)$, если $p=+\infty$ и пространство $H^{1}\left(\Omega, \Gamma_{1}\right)$, если $p<+\infty$. Положим $R_{\varepsilon} f^{0}=f^{0}$ для любого $f^{0} \in H_{0}$.

Если $f^{0} \in V$, то

$$
\begin{aligned}
\left(R_{\varepsilon} f^{0}, R_{\varepsilon} f^{0}\right)_{H_{\varepsilon}} & =\int_{\Omega} \rho^{\varepsilon}(x)\left(f^{0}\right)^{2} d x=\int_{\Omega \backslash B_{\varepsilon}}\left(f^{0}\right)^{2} d x+(\varepsilon \delta)^{-m} \int_{B_{\varepsilon}}\left(f^{0}\right)^{2} d x \\
& \rightarrow \int_{\Omega}\left(f^{0}\right)^{2} d x=\left(f^{0}, f^{0}\right)_{H} \quad \text { при } \quad \varepsilon \rightarrow 0
\end{aligned}
$$

в силу леммы 2. Действительно,

$$
\lim _{\varepsilon \rightarrow 0}(\varepsilon \delta)^{-m} \int_{B_{\varepsilon}}\left(f^{0}\right)^{2} d x=\lim _{\varepsilon \rightarrow 0}(\varepsilon \delta)^{2-m} \int_{B_{\varepsilon}}\left(f^{0}\right)^{2}(\varepsilon \delta)^{-2} d x
$$

HO

$$
\lim _{\varepsilon \rightarrow 0}(\varepsilon \delta)^{-2} \int_{B_{\varepsilon}}\left(f^{0}\right)^{2} d x=0,
$$

a $m<2$. Это означает, что справедливо условие С1 при $\varkappa=1$. 
Обозначим через $A_{\varepsilon}: H_{\varepsilon} \rightarrow H_{\varepsilon}$ оператор, ставящий в соответствие функции $f^{\varepsilon} \in H_{\varepsilon}$ решение $u^{\varepsilon} \in H^{1}\left(\Omega, \gamma_{\varepsilon}\right)$ задачи (4). Через $A_{0}: H_{0} \rightarrow H_{0}$ обозначим оператор, переводящий $f^{0} \in H_{0}$ в решение $u^{0} \in H^{1}\left(\Omega, \Gamma_{1}\right)$ задачи (5).

Легко проверить, что операторы $A_{\varepsilon}$ и $A_{0}$ являются положительньми компактными и самосопряженными в пространствах $H_{\varepsilon}$ и $H_{0}$ соответственно (см. [36]). Равномерная ограниченность семейства операторов в соответствующей операторной норме

$$
\sup _{\varepsilon}\left\|A_{\varepsilon}\right\|_{\mathscr{L}\left(H_{\varepsilon}\right)}<M
$$

следует из леммы 3 , поскольку при $m<2$ в силу лемм 1 и 3 , а также неравенства типа фидрихса

$$
\begin{aligned}
\left\|A_{\varepsilon} f^{\varepsilon}\right\|_{H_{\varepsilon}}^{2} & =\int_{\Omega} \rho^{\varepsilon}(x)\left(u^{\varepsilon}\right)^{2} d x \\
& \leqslant\left(\int_{\Omega \backslash B_{\varepsilon}}\left(u^{\varepsilon}\right)^{2} d x+(\varepsilon \delta)^{-2} \int_{B_{\varepsilon}}\left(u^{\varepsilon}\right)^{2} d x\right) \leqslant C_{4} \int_{\Omega}\left|\nabla u^{\varepsilon}\right|^{2} d x \\
& \leqslant C_{5}\left((\varepsilon \delta)^{2-m} \int_{B_{\varepsilon}} \rho^{\varepsilon}(x)\left(f^{\varepsilon}\right)^{2} d x+\int_{\Omega \backslash B_{\varepsilon}} \rho^{\varepsilon}(x)\left(f^{\varepsilon}\right)^{2} d x+\int_{B_{\varepsilon}}\left(f^{\varepsilon}\right)^{2} d x\right) \\
& \leqslant C_{6}\left(\int_{\Omega \backslash B_{\varepsilon}} \rho^{\varepsilon}(x)\left(f^{\varepsilon}\right)^{2} d x+\int_{B_{\varepsilon}} \rho^{\varepsilon}(x)\left(f^{\varepsilon}\right)^{2} d x\right)=C_{6}\left\|f^{\varepsilon}\right\|_{H_{\varepsilon}}^{2} .
\end{aligned}
$$

Таким образом, условие С2 имеет место.

Докажем выполнение условия С3. Пусть $f^{0} \in H_{0}$. Тогда

$$
A_{\varepsilon} R_{\varepsilon} f^{0}=u^{\varepsilon}, \quad R_{\varepsilon} A_{0} f^{0}=u^{0}
$$

где

$$
\begin{aligned}
& \Delta u^{\varepsilon}=-\rho^{\varepsilon}(x) f^{0} \quad \text { в } \Omega, \quad u^{\varepsilon} \in H^{1}\left(\Omega, \gamma_{\varepsilon}\right), \\
& \Delta u^{0}=-f^{0} \quad \text { в } \Omega, \quad u^{0} \in H^{1}\left(\Omega, \Gamma_{1}\right) .
\end{aligned}
$$

Для простоты будем считать, что $f^{\varepsilon} \equiv f^{0} \equiv f$. Хотя в случае, когда $f^{\varepsilon} \rightarrow f^{0}$ в соответствующем смысле, задача может быть изучена аналогично. В дальнейшем предполагается, что $f \in L_{\infty}(\Omega)$. Если $u^{0} \in H^{1}(\Omega)$ - обобщенное решение задачи (5), то в силу регулярности решений эллиптических уравнений [46] $u^{0} \in W_{l}^{2}(\Omega)$ для любого $l>1$. В силу теорем вложения [47]-[49] отсюда следует, что $u^{0}, \nabla u^{0} \in L_{\infty}(\Omega)$. Из первого уравнения задачи (5) следует, что $\Delta u^{0} \in L_{\infty}(\Omega)$, причем

$$
\left\|u^{0}\right\|_{L_{\infty}(\Omega)}+\left\|\nabla u^{0}\right\|_{L_{\infty}(\Omega)}+\left\|\Delta u^{0}\right\|_{L_{\infty}(\Omega)} \leqslant \text { const }\|f\|_{L_{\infty}(\Omega)} .
$$

Аналогичные оценки для $f \in L_{2}(\Omega)$ могут быть также получены (см., например, [34] и [50]).

Слабая сходимость $u^{\varepsilon}$ к $u^{0}$ в $H^{1}(\Omega)$ при $\varepsilon \rightarrow 0$ доказана в [36, теорема 1$]$, а также см. [22].

Напомним, что

$$
p:=\lim _{\varepsilon \rightarrow 0} \frac{\varepsilon^{n-2} \sigma_{n} c_{\gamma_{0}} / 2}{\delta(\varepsilon)} .
$$


Теорема 3. Если $p<+\infty, u^{\varepsilon} u u^{0}$ - обобщенные решения задач (4) $и$ (5) соответственно, то существует такая константа $K_{3}$, не зависящая от $\varepsilon, \delta, f$, что для достаточно малых $\varepsilon$ имеем

$$
\left\|u^{0} w_{\varepsilon}-u^{\varepsilon}\right\|_{H^{1}(\Omega)} \leqslant K_{3}\|f\|_{L_{\infty}(\Omega)}\left(\left(\nu_{\varepsilon}\right)^{1 / 2}+\left|\frac{\nu_{\varepsilon}}{\delta}-p\right|+(\varepsilon \delta)^{2-m}\right) .
$$

ДоКАЗАТЕЛЬСТво. Имея в виду свойства функции $u^{0}, w_{\varepsilon}$ и $u^{\varepsilon}$, получаем следующее интегральное выражение для любого $v \in H^{1}\left(\Omega, \gamma_{\varepsilon}\right)$ :

$$
\begin{gathered}
\int_{\Omega}\left(\nabla\left(u^{0} w_{\varepsilon}-u^{\varepsilon}\right), \nabla v\right) d x=\int_{\Omega}\left(w_{\varepsilon}-1\right)\left(2 \Delta u^{0} v+2\left(\nabla v, \nabla u^{0}\right)+\rho^{\varepsilon} f v\right) d x \\
+\left|\frac{\nu_{\varepsilon}}{\delta}-p\right| \int_{\partial \Omega} u^{0} w_{\varepsilon} v d s+2 \int_{\partial \Omega}\left(w_{\varepsilon}-1\right) p u^{0} v d s-\int_{\Omega} u^{0} \Delta w_{\varepsilon} v d x .
\end{gathered}
$$

Оценим интегралы, стоящие в правой части равенства. Имеем

$$
\begin{aligned}
& \left|\int_{\Omega}\left(w_{\varepsilon}-1\right)\left(2 \Delta u^{0} v+2\left(\nabla v, \nabla u^{0}\right)+\rho^{\varepsilon} f v\right) d x\right| \\
& \leqslant\left|\int_{\Omega} 2\left(w_{\varepsilon}-1\right) \Delta u^{0} v d x\right|+\left|\int_{\Omega} 2\left(w_{\varepsilon}-1\right)\left(\nabla v, \nabla u^{0}\right) d x\right| \\
& \quad+\left|\int_{\Omega \backslash B_{\varepsilon}}\left(w_{\varepsilon}-1\right) f v d x\right|+\left|\int_{B_{\varepsilon}}\left(w_{\varepsilon}-1\right)(\varepsilon \delta)^{-m} f v d x\right| \\
& \quad \leqslant\left\|w_{\varepsilon}-1\right\|_{L_{2}(\Omega)}\left(\left(\int_{\Omega} 4\left(\Delta u^{0} v\right)^{2} d x\right)^{1 / 2}+\left(\int_{\Omega} 4\left(\nabla u^{0}, \nabla v\right)^{2} d x\right)^{1 / 2}\right. \\
& \left.\quad+\left(\int_{\Omega \backslash B_{\varepsilon}}(f v)^{2} d x\right)^{1 / 2}\right)+(\varepsilon \delta)^{2-m}\|v\|_{H^{1}(\Omega)}\|f\|_{L_{\infty}(\Omega)} \\
& \leqslant C_{7}\left(\nu_{\varepsilon} \delta\right)^{1 / 2}\|f\|_{L_{\infty}(\Omega)}\|v\|_{H^{1}(\Omega)}+(\varepsilon \delta)^{2-m}\|f\|_{L_{\infty}(\Omega)}\|v\|_{H^{1}(\Omega)} .
\end{aligned}
$$

Здесь была использована лемма 1 и свойства функции $w_{\varepsilon}$. Из свойства функций $u^{0}$ и $w_{\varepsilon}$ следуют оценки

$$
\begin{gathered}
\left|\int_{\Omega} u^{0} \Delta w_{\varepsilon} v d x\right| \leqslant C_{8}\left(\nu_{\varepsilon} \delta\right)^{1 / 2}\|v\|_{H^{1}(\Omega)}\|f\|_{L_{\infty}(\Omega)}, \\
\left|\frac{\nu_{\varepsilon}}{\delta}-p\right|\left|\int_{\partial \Omega} u^{0} w_{\varepsilon} v d s\right| \leqslant C_{9}\left|\frac{\nu_{\varepsilon}}{\delta}-p\right|\|v\|_{H^{1}(\Omega)}\|f\|_{L_{\infty}(\Omega)} .
\end{gathered}
$$

Оставшийся интеграл оценивается следуюшим образом:

$$
\left|\int_{\partial \Omega}\left(w_{\varepsilon}-1\right) p u^{0} v d s\right| \leqslant C_{10}\left(\nu_{\varepsilon}\right)^{1 / 2}\|v\|_{H^{1}(\Omega)}\|f\|_{L_{\infty}(\Omega)} .
$$

Подставляя $v=u^{0} w_{\varepsilon}-u^{\varepsilon}$ и используя лемму 2 , получаем окончательную оценку

$$
\left\|u^{0} w_{\varepsilon}-u^{\varepsilon}\right\|_{H^{1}(\Omega)} \leqslant K_{3}\|f\|_{L_{\infty}(\Omega)}\left(\left(\nu_{\varepsilon}\right)^{1 / 2}+\left|\frac{\nu_{\varepsilon}}{\delta}-p\right|+(\varepsilon \delta)^{2-m}\right) .
$$

Теорема доказана. 
Tеорема 4. Eсли $p=+\infty$, то существует $K_{4}$ maкое, что

$$
\left\|u^{0} w_{\varepsilon}-u^{\varepsilon}\right\|_{H^{1}(\Omega)} \leqslant K_{4}\|f\|_{L_{\infty}(\Omega)}\left(\left(\nu_{\varepsilon}\right)^{1 / 2}+\left(\frac{\delta}{\nu_{\varepsilon}}\right)^{1 / 2}+(\varepsilon \delta)^{2-m}\right) .
$$

ДоКАЗАТЕЛЬСТВо проводится аналогично с использованием результатов [36].

Продолжим проверку условия С3. Пользуясь леммой 1 и неравенством Фридрихса, устанавливаем

$$
\begin{aligned}
& \left\|A_{\varepsilon} R_{\varepsilon} f^{0}-R_{\varepsilon} A_{0} f^{0}\right\|_{H_{\varepsilon}}^{2}=\int_{\Omega} \rho^{\varepsilon}(x)\left|u^{\varepsilon}-u^{0}\right|^{2} d x \\
& \quad \leqslant \int_{\Omega \backslash B_{\varepsilon}}\left|u^{\varepsilon}-u^{0}\right|^{2} d x+\int_{B_{\varepsilon}}(\varepsilon \delta)^{-m}\left|u^{\varepsilon}-u^{0}\right|^{2} d x \\
& \quad \leqslant C_{11} \int_{\Omega}\left|\nabla\left(u^{\varepsilon}-u^{0}\right)\right|^{2} d x+C_{12}(\varepsilon \delta)^{2-m} \int_{B_{\varepsilon}}\left|\nabla\left(u^{\varepsilon}-u^{0}\right)\right|^{2} d x \\
& \quad \leqslant C_{13} \int_{\Omega}\left|\nabla\left(u^{\varepsilon}-u^{0}\right)\right|^{2} d x \\
& \quad \leqslant \int_{\Omega}\left|\nabla\left(u^{\varepsilon}-u^{0} w_{\varepsilon}\right)\right|^{2} d x+\int_{\Omega}\left(\left|\nabla u^{0}\right|^{2}\left(1-w_{\varepsilon}\right)^{2}+\left(u^{0}\right)^{2}\left|\nabla\left(1-w_{\varepsilon}\right)\right|^{2}\right) d x .
\end{aligned}
$$

С учетом свойств функций $u^{0}$ и $w_{\varepsilon}$, а также теорем 3,4 и леммы 2 при $m<2$, мы получаем условие С3.

Докажем справедливость условия С4.

Если $\sup _{\varepsilon}\left\|f^{\varepsilon}\right\|_{H \varepsilon}<\infty$, то из леммы 3 и неравенства Фридрихса вытекает, что

$$
\sup _{\varepsilon}\left\|u^{\varepsilon}\right\|_{H^{1}\left(\Omega, \gamma_{\varepsilon}\right)}<\infty
$$

где $u^{\varepsilon}$ - решение задачи (4). Следовательно (по теореме Реллиха), существует $U^{*} \in V$ и подпоследовательность $\varepsilon^{\prime} \rightarrow 0$ такие, что

$$
U^{\varepsilon^{\prime}} \rightarrow U^{*} \quad \text { слабо в } H^{1}(\Omega) \text { и сильно в } L_{2}(\Omega) .
$$

Поэтому, пользуясь леммой 1 , выводим

$$
\begin{aligned}
\left\|A_{\varepsilon} f^{\varepsilon}-R_{\varepsilon} U^{*}\right\|_{H_{\varepsilon}}^{2} & =\int_{\Omega} \rho^{\varepsilon}(x)\left|u^{\varepsilon}-U^{*}\right|^{2} d x \\
& \leqslant \int_{\Omega \backslash B_{\varepsilon}}\left|u^{\varepsilon}-U^{*}\right|^{2} d x+(\varepsilon \delta)^{-m} \int_{B_{\varepsilon}}\left|u^{\varepsilon}-U^{*}\right|^{2} d x \\
& \leqslant \int_{\Omega}\left|u^{\varepsilon}-U^{*}\right|^{2} d x+C_{14}(\varepsilon \delta)^{2-m} \int_{\Omega}\left|\nabla\left(u^{\varepsilon}-U^{*}\right)\right|^{2} d x
\end{aligned}
$$

где $u^{\varepsilon}=A_{\varepsilon} f^{\varepsilon}$ и $C_{14}$ не зависит от $\varepsilon$. Отсюда получаем соотношение из условия С4, так как $m<2$ и имеет место сходимость (14).

Таким образом, выполнены условия С1-C4 и мы можем применить теорему о сходимости спектров последовательности операторов, заданных в различных гильбертовых пространствах (Олейник-Иосифьяна-Шамаева).

Задача на собственные значения для оператора $A_{0}$ имеет вид (12), где $\mu_{0}^{k}=1 / \lambda_{0}^{k}$, а $\lambda_{0}^{k}$ - собственные значения задачи (6).

Итак, имеет место 
ТЕОрема 5. Пусть $\lambda_{0}^{k}, \lambda_{\varepsilon}^{k}$ являются собственными значениями задач (6) $и$ (1) соответственно. Тогда

$$
\begin{array}{ll}
\left|\lambda_{0}^{k}-\lambda_{\varepsilon}^{k}\right| \leqslant C_{k}^{3}\left(\left(\nu_{\varepsilon}\right)^{1 / 2}+(\varepsilon \delta)^{2-m}+\left|\frac{\nu_{\varepsilon}}{\delta}-p\right|\right), & \text { eсли } \quad p<\infty, \\
\left|\lambda_{0}^{k}-\lambda_{\varepsilon}^{k}\right| \leqslant C_{k}^{4}\left(\left(\nu_{\varepsilon}\right)^{1 / 2}+\left(\frac{\delta}{\nu_{\varepsilon}}\right)^{1 / 2}+(\varepsilon \delta)^{2-m}\right), \quad \text { eсли } \quad p=+\infty
\end{array}
$$

где постоянные $C_{k}^{3}, C_{k}^{4}$ не зависят от $\varepsilon$.

Если кратность собственного значения $\lambda_{0}^{l}$ задачи (6) равна $r$, m.e. $\lambda_{0}^{l}=\lambda_{0}^{l+1}=$ $\cdots=\lambda_{0}^{l+r}$, то для любой собственной функиии и $u_{0}^{l}$ задачи (6), соответствующей собственному значению $\lambda_{0}^{l},\left\|u_{0}\right\|_{L_{2}(\Omega)}=1$, существует линейная комбинация $\overline{u^{\varepsilon}}$ собственных функиий задачи (1), соответствующих собственному значению $\lambda_{\varepsilon}^{l+1}, \ldots, \lambda_{\varepsilon}^{l+r}$ такая, что

$$
\begin{aligned}
& \left(\int_{\Omega} \rho^{\varepsilon}(x)\left|\overline{u^{\varepsilon}}-u_{0}^{l}\right|^{2} d x\right)^{1 / 2} \leqslant C_{l}^{3}\left(\left(\nu_{\varepsilon}\right)^{1 / 2}+(\varepsilon \delta)^{2-m}+\left|\frac{\nu_{\varepsilon}}{\delta}-p\right|\right), \quad \text { ecлu } \quad p<\infty \\
& \left(\int_{\Omega} \rho^{\varepsilon}(x)\left|\overline{u^{\varepsilon}}-u_{0}^{l}\right|^{2} d x\right)^{1 / 2} \leqslant C_{l}^{4}\left(\left(\nu_{\varepsilon}\right)^{1 / 2}+\left(\frac{\delta}{\nu_{\varepsilon}}\right)^{1 / 2}+(\varepsilon \delta)^{2-m}\right), \quad \text { ecлu } \quad p=+\infty .
\end{aligned}
$$

әде постоянные $C_{l}^{3}, C_{l}^{4}$ не зависят от в и $u_{0}^{l}$.

Используя предложение 1, из теорем 3-5 можно легко вывести утверждения теорем 1 и 2 .

Благодарности. В основном статья была написана летом 1994 года в г. Севастополе, а окончательный вариант подготовлен в г. Нарвике на севере Норвегии зимой 2003 года. Автор выражает признательность Университетскому колледжу Нарвика (Narvik University College) за прекрасные условия для работы.

\section{СПИСОК ЦИТИРОВАННОЙ ЛИТЕРАТУРЫ}

[1] Крылов А.Н. О некоторых дифференциальных уравнениях математической физики, имеющих приложения в технических вопросах // Изв. Николаевской морской академии. 1913. Вып. 2. С. 325-348.

[2] Тихонов А. Н., Самарский А. А. Уравнения математической физики. М.: Наука, 1972.

[3] Sanchez-Palencia É. Perturbation of eigenvalues in thermoelasticity and vibration of a system with concentrated masses / / Trends and Application of Pure Math. to Mechanics. Lecture Notes in Phisics. V. 195. Berlin: Springer-Verlag, 1984. P. 346-368.

[4] Олейник О. А. Лекции по уравнениям с частными производными. М.: Изд-во МГУ, 1976.

[5] Олейник О.А. О собственных колебаниях тел с концентрированными массами // Современные проблемы прикладной математики и математической физики. М.: Наука, 1988. С. $101-128$.

[6] Олейник О. А. О спектрах некоторых сингулярно возмущенных операторов // УМН. 1987. T. 42. № 3. C. 221-222.

[7] Oleinik O. A. Homogenization problems in elasticity. Spectrum of singularly perturbed operators // Lecture Notes Series. V. 122. Cambridge: Cambridge University Press, 1987. P. 188-205. 
[8] Олейник О.А. О частотах собственных колебаний тел с концентрированными массами // Функциональные и численные методы математической физики. Киев: Наукова думка, 1988. C. $165-171$.

[9] Головатый Ю. Д. Спектральные свойства колебательных систем с присоединенными массами. Дисс. ... к.ф.-м.н. М.: МГУ, 1988.

[10] Головатый Ю. Д., Назаров С. А., Олейник О. А., Соболева Т. С. О собственных колебаниях струны с присоединенной массой // Сиб. матем. ж. 1988. Т. 29. № 5. С. 71-91.

[11] Олейник О.А., Соболева Т. С. О собственных колебаниях неоднородной струны с конечным числом присоединенных масс // УМН. 1988. Т. 43. № 4. С. 187-188.

[12] Головатый Ю. Д. О собственных колебаниях и собственных частотах упругого стержня с присоединенной массой // УМН. 1988. Т. 43. № 4. С. 173-174.

[13] Головатый Ю. Д. О собственных колебаниях и собственных частотах закрепленной пластинки с присоединенной массой // УМН. 1988. Т. 43. № 5. С. 185-186.

[14] Головатый Ю. Д. Спектральная задача Неймана для оператора Лапласа с сингулярно возмущенной плотностью // УМН. 1990. Т. 45. № 4. С. 147-148.

[15] Назаров С. А. Об одной задаче Санчес-Паленсия с краевыми условиями Неймана // Изв. вузов. Матем. 1989. № 11. С. 60-66.

[16] Рахманов Н. У. О собственных колебаниях систем с концентрированными массами. Дисс. ‥к.ф.-м.н. М.: МГУ, 1991.

[17] Головатый Ю. Д., Назаров С. А., Олейник О. А. Асимптотика собственных значений и собственных функций в задачах о колебаниях среды с сингулярньм возмущением плотности // УМН. 1988. Т. 43. № 5. С. 189-190.

[18] Головатый Ю. Д., Назаров С. А., Олейник О.А. Асимптотические разложения собственных значений и собственных функций задач о колебаниях среды с концентрированными возмущениями // Тр. МИАН. 1990. Т. 192. С. 42-60.

[19] Sanchez-Palencia É., Tchatat H. Vibration de systèmes elastiques avec des masses concentrées // Rend. Sem. Mat. Univ. e Politecnico di Torino. 1984. V. 42. № 3. P. 43-63.

[20] Leal C., Sanchez-Hubert J. Perturbation of the eigenvalue of a membrane with a concentrated mass // Quart. Appl. Math. 1989. V. 47. №1. P. 93-103.

[21] Lobo M., Pérez E. Asymptotic behavior of the vibrations of a body having many concentrated masses near the boundary // C. R. Acad. Sci. Paris. Sér. II. 1992. V. 314. P. 13-18.

[22] Lobo M., Pérez E. On vibrations of a body with many concentrated masses near the boundary // Math. Models and Methods in Appl. Sci. 1993. V. 3. № 2. P. 249-273.

[23] Lobo M., Pérez E. Vibrations of a body with many concentrated masses near the boundary: high frequency vibrations // Spectral Analysis of Complex Structures. Paris: Hermann, 1995. P. 85-101.

[24] Lobo M., Pérez E. Vibrations of a membrane with many concentrated masses near the boundary // Math. Models and Methods in Appl. Sci. 1995. V. 5. № 5. P. 565-585.

[25] Lobo M., Pérez E. High frequency vibrations in a stiff problem // Math. Models and Methods in Appl. Sci. 1997. V. 7. № 2. P. 291-311.

[26] Lobo M., Pérez E. A skin effect for systems with many concentrated masses // C. R. Acad. Sci. Paris. Sér. IIb. 1999. V. 327. P. 771-776.

[27] Gómez D., Lobo M., Pérez E. On the eigenfunctions associated with the high frequencies in systems with a concentrated mass // J. Math. Pures Appl. 1999. V. 78. P. 841-865.

[28] Lobo M., Pérez E. The skin effect in vibrating systems with many concentrated masses // Math. Methods Appl. Sci. 2001. V. 24. №1. P. 59-80.

[29] Oleinik O. A., Sanchez-Hubert J., Yosifian G. A. On vibration of membrane with concentrated masses // Bull. Sci. Math. 1991. V. 115. № 1. P. 1-27.

[30] Sanchez-Hubert J., Sanchez-Palencia É. Vibration and Coupling of Continuous System. Asymptotic methods. Berlin-Heidelberg: Springer-Verlag, 1989. 
[31] Sanchez-Hubert J. Perturbation des valeurs propres pour des systèmes avec masse concentrée // C. R. Acad. Sci. Paris Ser. II Mec. Phys. Chim. Sci. Univers Sci. Terre. 1989. V. 309. №6. P. 507-510.

[32] Доронина Е.И., Чечкин Г.А. О собственных колебаниях тела с большим количеством непериодически расположенных концентрированных масс // Тр. МИАН. 2002. Т. 236. C. $158-166$.

[33] Rybalko V. Vibration of elastic systems with a large number of tiny heavy inclusions // Asymptotic Analysis. 2002. V. 32. № 1. P. 27-62.

[34] Перес М.Е., Чечкин Г. А., Яблокова (Доронина) Е. И. О собственных колебаниях тела с "легкими" концентрированньми массами на поверхности // УМН. 2002. Т. 57. №6. C. $195-196$.

[35] Чечкин Г. А. О колебаниях тел с концентрированньми массами, расположенными на границе // УМН. 1995. Т. 50. № 4. С. 105-106.

[36] Чечкин Г.А. Усреднение краевых задач с сингулярным возмущением граничных условий // Матем. сб. 1993. Т. 184. №6. С. 99-150.

[37] Iosida K. Functional Analysis. New York: Springer-Verlag, 1965.

[38] Гадыльшин Р. Р., Чечкин Г. А. Краевая задача для Лапласиана с быстро меняющимся типом граничных условий в многомерной области // Сиб. матем. ж. 1999. Т. 40. № 2 . C. 271-287.

[39] Кондратьев В. А., Олейник О.А. Об асимптотике в окрестности бесконечности решений с конечным интегралом Дирихле для эллиптических уравнений второго порядка // Тр. семинара им. И. Г. Петровского. Т. 12. М.: Изд-во МГУ, 1987. С. 149-163.

[40] Kondratiev V. A., Oleinik O. A. Asymptotic properties of the elasticity system // Proceedings of the International Conference "Application of Multiple Scaling in Mechanics". Paris: Masson, 1987. P. 188-205.

[41] Олейник О. А., Иосифьян Г. А., Шамаев А. С. Математические задачи теории сильно неоднородных упругих сред. М.: МГУ, 1990.

[42] Олейник О. А., Чечкин Г. А. О краевых задачах для эллиптических уравнений с быстро меняющимся типом граничных условий // УМН. 1993. Т. 48. №6. С. 163-164.

[43] Oleinik O. A., Chechkin G. A. On Asymptotics of Solutions and Eigenvalues of an Elliptic Problem with Rapidly Alternating Type of Boundary Conditions. Trieste: International Center for Theoretical Physics, SMR 719/4, 1993.

[44] Олейник О.А., Чечкин Г. А. Об одной задаче граничного усреднения для системы теории упругости // УМН. 1994. Т. 49. № 4. С. 114.

[45] Oleinik O. A., Chechkin G. A. Solutions and eigenvalues of the boundary value problems with rapidly alternating boundary conditions for the system of elasticity. // Rend. Lincei Math. Appl. (9). 1996. V. 7. №1. P. 5-15.

[46] Агмон С., Дуглис А., Ниренберг Л. Оценки решений эллиптических уравнений вблизи границы. М.: ИЛ, 1962.

[47] Ладыженская О. А. Краевые задачи математической физики. М.: Наука, 1973.

[48] Соболев С. Л . Некоторые применения функционального анализа в математической физике. М.: Наука, 1988.

[49] Соболев С. Л. Избранные вопросы теории функциональных пространств и обобщенных функций. М.: Наука, 1989.

[50] Chechkin G. A., Pérez M. E., Yablokova E. I. On eigenvibrations of a body with many "light" concentrated masses located nonperiodically along the boundary // Preprint of Universidad de Cantabria Núm. 1/2002. Santander, Abril 2002. 